\title{
AugerPrime Upgraded Electronics
}

\author{
G. Marsella ${ }^{a, b, *}$ on behalf of the Pierre Auger ${ }^{c}$ Collaboration \\ (a complete list of authors can be found at the end of the proceedings) \\ a Università degli Studi di Palermo, Dipartimento di Fisica e Chimica "E. Segrè" \\ Via delle Scienze ed. 17, 90128 Palermo, Italy \\ ${ }^{b}$ INFN Sezione Catania, \\ Via S. Sofia, 64, 95125 Catania, Italy \\ ${ }^{c}$ Observatorio Pierre Auger, \\ A. San Martín Norte 304, 5613 Malarg ue, Argentina \\ E-mail: spokespersons@auger.org
}

Since 2015, the Pierre Auger Observatory has been undergoing an important upgrade. It consists of the addition of Scintillator Surface Detectors (SSD) on top of the existing Water-Cherenkov Detectors (WCDs), as well as a small Photo-Multiplier Tube (sPMT) inside the WCDs (both excluding the outer crown of the array), an Underground Muon Detectors (UMD) in the limited higher density area, denominated Infill, where the stations are installed at a distance of $750 \mathrm{~m}$ instead of the standard $1.5 \mathrm{~km}$ (SD750 and later also in the SD433), and a Radio Detector antenna array (RD) at each of the 1660 surface detector stations. To process the signals of all these detector systems and to increase the dynamic range and time resolution, new electronics, Upgraded Unified Boards (UUBs) have been developed and are being produced and deployed at the Observatory. The combination of all of these new features of the Surface Detector (SD) will provide an enhanced capability for answering the still many open questions related to the nature of ultra-high energy cosmic rays.

In this work the main characteristics, the production and validation chain, the performances and the status of the implementation of the new Upgraded Unified Boards will be illustrated. The first data collected from the already operational upgraded stations in the array will also be presented.

$37^{\text {th }}$ International Cosmic Ray Conference (ICRC 2021)

July 12 th - 23rd, 2021

Online - Berlin, Germany

\footnotetext{
*Presenter
} 


\section{Introduction}

Since the beginning, the Pierre Auger Observatory[1] gave a strong contribution to the understanding of ultra high-energy cosmic rays (UHECRs). The principal motivation for the upgrade of the Pierre Auger Observatory, AugerPrime[2], is to provide additional measurements to address the following questions:

- The origin of the flux suppression at the highest energies and the measurement of the mass composition beyond the reach of the Fluorescence Detector (FD).

- Search for a proton contribution in the flux suppression region $\left(E>5 \times 10^{19} \mathrm{eV}\right)$, aiming at reaching a sensitivity to a proton contribution as small as $10 \%$ in this region, search of point sources and estimation of the physics potential of existing and future cosmic ray, neutrino, and gamma-ray experiments.

- Fundamental particle physics at energies beyond man-made accelerators and studies of extensive air showers and hadronic multiparticle production.

The proposed upgrade consist of: (i) the addition of a plastic scintillator plane (SSD[3]) above the existing Water-Cherenkov Detectors (WCD); (ii) the installation in the Surface Detector (SD) stations of new electronics that will process the signals from all AugerPrime detectors; (iii) the installation of an additional small PMT (sPMT[4]) in the WCD to increase the dynamic range, (iv) the installation of an underground muon detector (UMD[5]) in the existing SD Infill area; (v) Radio Detector $(\mathrm{RD}[6])$ added on top of each water Cherenkov detector. AugerPrime is in the deployment phase for the various detectors. The electronics entered in the production phase. Already 81 boards are in the field; 84 new boards will be sent to Argentina at the end of July 2021, 400 by the end of August.

\section{The Upgraded Unified Boards}

The new electronics[7] processes the signals of SSD, SPMT, interfaces UMD and RD and provides a faster sampling of ADC traces for the WCD PMTs. The original Unified Board (UB) will be replaced by the UUB, which is designed to fit the existing enclosure, and accept the existing PMT, GPS antenna, and communication cables. The new electronics implements faster ADCs (120 MHz instead of $40 \mathrm{MHz}$ ) with larger dynamic range (12 bits each instead of 10 bits) and a significantly more powerful FPGA. Specifically, it employs a Xilinx Zync-7020 Programmable SoC (Artix-7 FPGA and associated Cortex A9 Dual 333 MHz ARM co-processor). The logic code of AugerPrime is primarily written in IEEE standard synthesizable Verilog. Xilinx Vivado is used for standard modules such as memories, UARTs, and processor bus interfaces. Xilinx PetaLinux, a light LINUX operative system, runs on the embedded ARM processor. The UUB integrates various functions (analog signal processing, triggering, calibration, GPS time tagging, and data acquisition) on a single board. The FPGA is connected to a 4 Gbit LP- DDR2 memory and 2 Gbit flash memory. The FPGA implements in programmable logic basic digital functions like the readout of the ADCs, the generation of triggers, and the interfaces to the LED flasher, GPS receiver, and memories. High-level functions like the data handling and the communications with the radio transmitter are 
implemented. The current local station software, originally written in C under OS-9, has been ported to PetaLinux operating system which expands the possible pool of experts for DAQ code development and maintenance. The speed of the upgraded CPU is more than 10 times faster than the previous one, with a similar increase in memory. This allows more sophisticated processing within the local station. The addition of accessible trigger IN/OUT signals, serial terminal, ethernet connection and high-speed USB port facilitates tests both in the lab and in the field.

\section{Production and Test}

The UUB are produced and assembled in Italy by SITAEL SpA ${ }^{1}$. The SlowControl (SC) and Zynq flash are programmed, heat sinks attached and conformal coating applied during production. All the UUBs have a record in the KIT database (containing the UUB production number and the internal serial number). After the validation with prototypes, a sample of pre-production UUBs are taking data since December 2020. The boards, before reaching the field, undergo 2 test phases[8]: 1) Manufacturer test directly in the company where the boards are realised;2) Environmental Stress Screening (ESS) tests realized in Prague by the local Auger group.
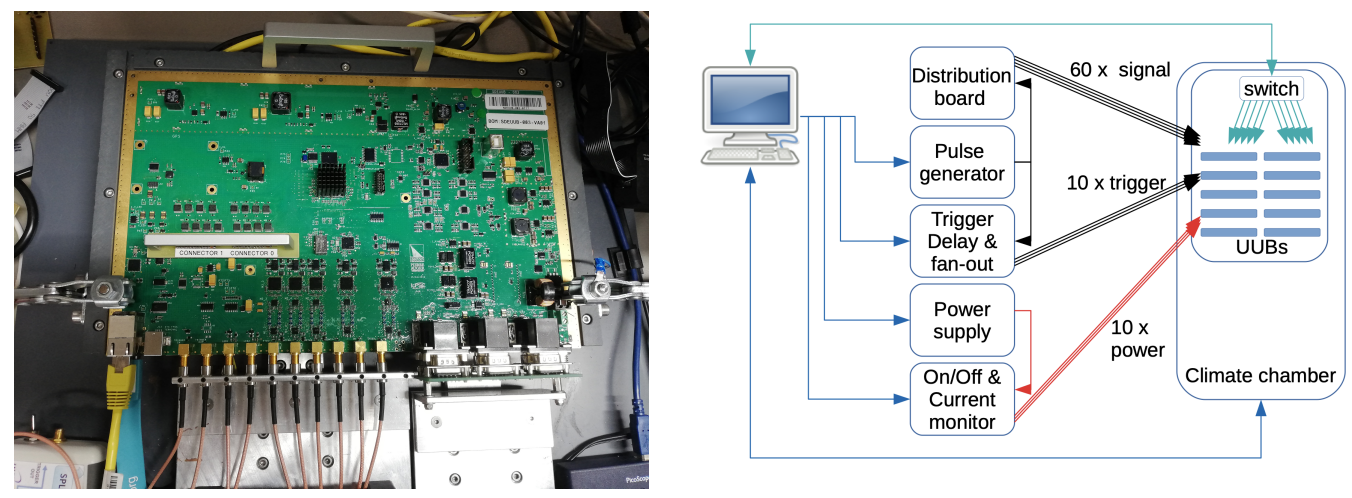

Figure 1: Left: UUB in Manufacturer Test system. Right: ESS Test system description.

\subsection{Manufacturer test}

The manufacturer test, setup in the producer SITAEL company, aims at verifying that all functional blocks of the UUBs are correctly assembled and in operation. The UUB is connected on a stable socket of $160 \mathrm{~cm} \times 80 \mathrm{~cm}$ size. The front-end is connected to a pulse generator while the GPS, HV control connectors of the PMTs and the digital interfaces are connected to loop-back adapters (Fig.1 (Left)).

As a first step of the testing procedure, all UUBs should pass an initial automated optical inspection. The inspection allows us to detect problems related to the soldering process (such as excessive or insufficient solder paste) and issues related to component assembly (such as missing components, wrong orientation or distortion of integrated circuits, wrong component polarity) with high efficiency.

\footnotetext{
${ }^{1}$ https://www.sitael.com
} 
Once the automatic procedure is complete, the operator moves the board to the semi-automatic test bench. After connecting all inputs to the test system a script is executed to install the proper operative system and execute the full functionality test. Through a web page it is possible to fill the requested information about components and execute specific tests using an Application Programming Interface (API) running under PetaLinux. After acquiring and analysing the test data, they are formatted and saved in a database to Karlsruhe Institute of Technology (KIT).

\subsection{Environmental Stress Screening test}

The UUBs are sent to the Institute of Physics, Prague after the manufacturing test. The handling of the UUBs follows all the ESD safe precautions foreseen by CEIEN 61340-5-1. The ESS testing is done in three steps: First, preliminary tests are executed to evaluate the noise and general status of each board individually. Next, 10 UUBs at a time are placed inside the climate chamber Binder MKFT115 at a minimum distance $5 \mathrm{~cm}$ from each other for the burn-in procedure. Finally, the Environmental Stress Screening is done in 10 cycles, going down to $-20^{\circ} \mathrm{C}$ and up to $+70^{\circ} \mathrm{C}$ (temperature change of $3^{\circ} \mathrm{C} / \mathrm{min}$ ). Thus, the measurement points are: $20^{\circ} \mathrm{C}, 0^{\circ} \mathrm{C},-20^{\circ} \mathrm{C}$, $0^{\circ} \mathrm{C}, 20^{\circ} \mathrm{C}, 45^{\circ} \mathrm{C}, 70^{\circ} \mathrm{C}$. At extreme temperatures the stabilisation and readout time take 15 minutes. During the two last cycles the power ON/OFF test is performed (over/under voltage protection). Further details on the tests performed can be found in [8].

The scheme of the ESS test bench is shown in Fig.1(Right). The communication with the boards is done via Ethernet connection through Mikrotik RB750r2 router placed inside the chamber. The test pulse is issued by AFG3252C (Tektronix), amplified/attenuated and distributed into 60 channels via a custom-made splitter unit. To power the boards, CPX400SP power supply is used, passing through another custom unit allowing monitoring of the current drawn by individual boards and switching the boards off one by one in case of a failure. This unit also provides signals to control the splitter. The last custom-made unit distributes the trigger signal to all ten boards using a suitable delay with respect to the pulse generator.

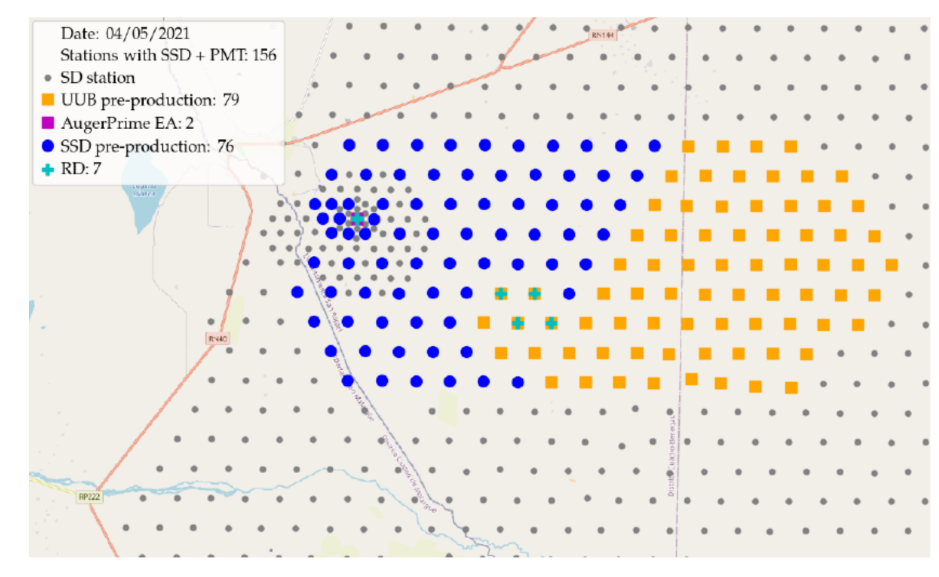

Figure 2: UUB pre-production array

The temperature inside the chamber is monitored by two independent temperature sensors and recorded every 30 seconds together with Zynq temperatures, SC values and the current drawn 
by each UUB. All Data are recorded into the official data-base sited in Prague and mirrored to Argentina where, after these tests, the board are sent to be assembled and deployed in the field.

\section{Deployment and Performances}

In Argentina, the boards undergo a final test before being fully integrated and installed in the field. Actually 81 boards are installed in the field (as shown in Fig.2), 79 in a pre-production engineering array and 2 in the AugerPrime stations Clais and Trak, the 2 stations where all the AugerPrime detectors are already installed and are used to test the AugerPrime integration.

Since the end of 2020, all installed boards are acquired with standard array and processed like old UBs. Currently the UUB trigger signals are filtered at $40 \mathrm{MHz}$ to emulate UB, in order to not perturbate the standard DAQ procedure, which in this phase is still dominated by the old electronics. Offline the UUB data are extracted for monitoring and testing purposes. The new devices such as SSD and SPMT, that can be acquired only by the new electronics, are then continuously studied by the collaboration. For each board, the ten ADC channels are verified to have stable baselines both in mean values and RMS. Also the calibration of all detectors are performed and monitored as shown in the following sections.

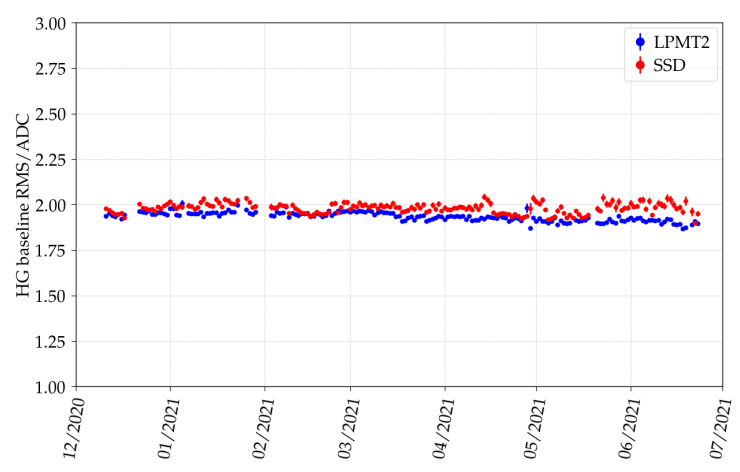

Figure 3: Distribution of the daily mean value of the baseline RMS distribution for the HG channel of a LPMT and an SSD channel. The error bar is the RMS of such daily distribution.

\subsection{WCD and SSD}

In the UUBs, the WCD PMT anode signal is divided in two channels: High Gain (HG) and Low Gain (LG). The HG/LG ratio is 32. The SSD LG channel is attenuated by a factor 4 to extend the dynamic range. So the SSD HG/LG ratio is 128. In Fig.3 the HG RMS of the baseline distribution in function of time is shown for station 22 with UUB implemented. A WCD LPMT and an SSD signals are shown. In all stations the HG channel RMS are less than 2 ADC counts while in LG channels are less than 0.5 ADC counts. The data shown have been taken between January the 1st 2021 and May the 31st 2021. Each point is the daily mean value of the baseline RMS distribution and the error bar is the RMS of such daily distribution.

Also the baseline values are monitored and are very stable (within \pm 1 ADC count). In Fig.4 the distribution of the daily mean value of the baseline RMS for the stations 20 and 22 are shown (Left station 20 and Right station 22 signals). For the Calibration of the WCD PMTs the muon 

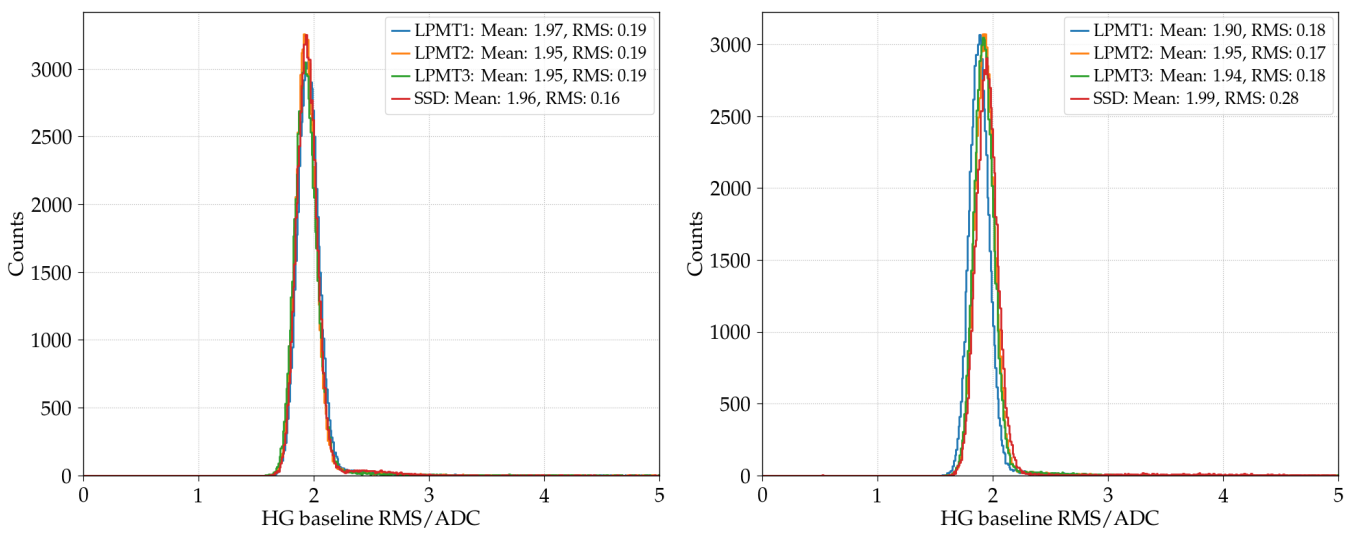

Figure 4: Distribution of the daily mean value of the baseline RMS for the stations 20 (Left) and 22 (Right)

signals are acquired by dedicated triggers and the Vertical Equivalent Muon (VEM) is evaluated using HG channel. The Low Gain channel is calibrated in the range of superposition of signals with HG. The SSD calibration is based on the signal of a minimum-ionizing particle (MIP) going through the detector. About $40 \%$ of the calibration triggers of the WCD produce a MIP in the SSD. We are studying the possibility to use SSD data to perform better WCD calibrations. An example of the VEM and MIP calibration histograms is shown in Fig.5. In this figure in the lower right plot the red line indicates the cut to apply on SSD ADC peak to enhance the hump to valley ratio for VEM evaluation in WCD PMTs calibration.

The time resolution has been studied with the prototype UUBs in two tanks (Clais and Trak). The result shows that, in measuring the fixed delay between these two stations with a mutual trigger, the time resolution is $\sim 5.0$ nanoseconds.

\subsection{The small PMT}

For the purpose of extending the dynamic range of the signal acquisition, the WCD is equipped with an additional small photomultiplier tube (sPMT), a 1" diameter Hamamatsu R8619, which signals are digitized with 12 bits at $120 \mathrm{MHz}$ in a separate channel. The sPMT features the same bialkali photocathode of the XP1850 model (WCD PMT) but with an active area 100 times smaller, potentially allowing for an equivalent dynamic range extension when operated at the same gain of the WCD PMTs $\left(\sim 3 \times 10^{5}\right)$. Adjusting the sPMT gain, the extension can be limited to a factor of $\sim 32$ allowing the measurement of signals up to the required target of 20,000 VEM without saturation.

Due to its small area, the sPMT cannot be calibrated using atmospheric muons. A dedicated selection of small local shower is set up to this aim, furthermore imposing a minimum threshold of 80 VEM on the WCD PMTs (corresponding to roughly 200 events per hour per station) to guarantee a reasonably large signal on the sPMT, not affected too much by statistical fluctuations. The signals in a region of superposition (which upper limit is given by the WCD PMTs saturation) are then exploited to cross-calibrate the sPMT using the VEM-calibrated signals of the WCD PMTs.

The sPMT cross-calibration factor is monitored and its value updated several times per day in the offline data analysis to follow the variation induced by the temperature dependence of the sPMT 

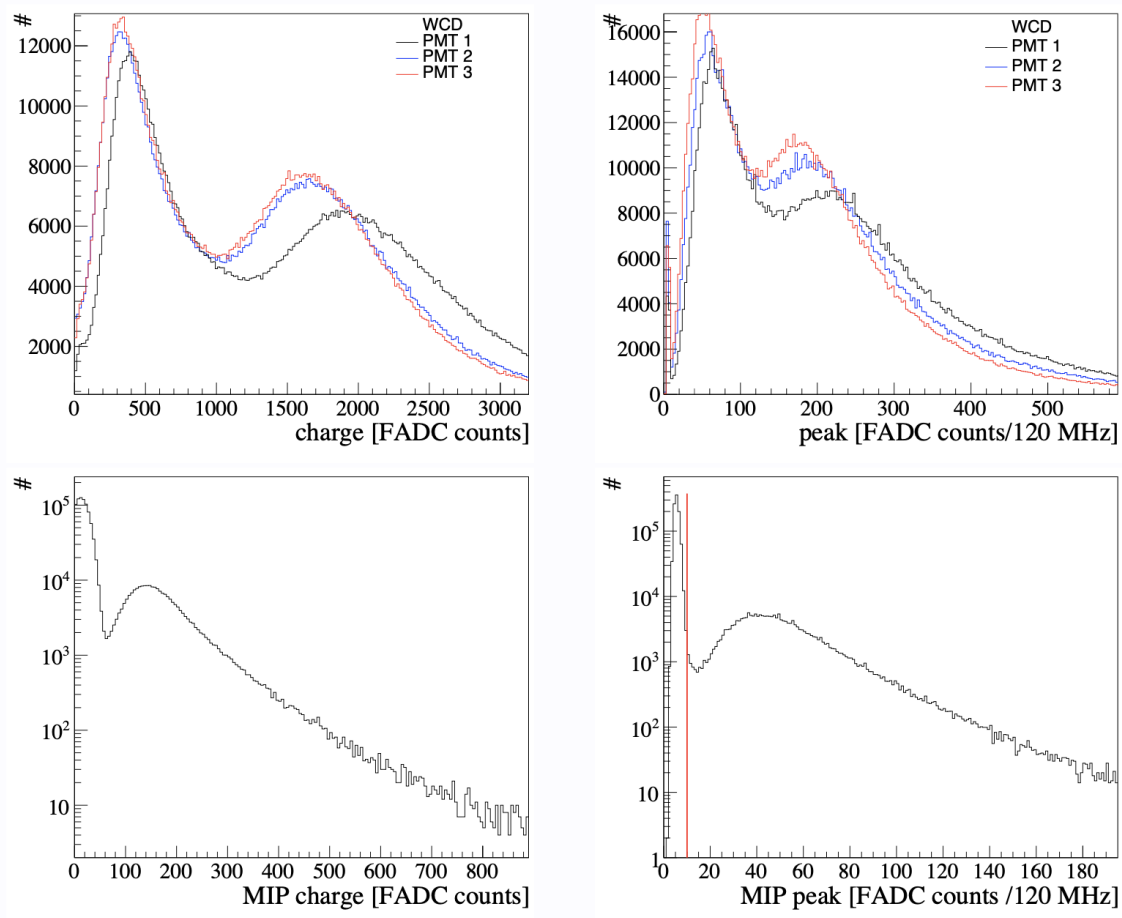

Figure 5: Calibration Histograms: Top - histograms show the muon charge (left) and peak (right) for the three WCD PMTs; Bottom - The muon charge (left) and peak (right) in the SSD. The red line in the last plot, indicates the cut to apply on SSD ADC peak to enhance the hump to valley ratio for VEM evaluation in WCD PMTs calibration

gain. The changes in the WCD PMTs gain is instead accounted for by continuously updating the single muon calibration.

The cross-calibration factor is also employed to estimate the maximum signal $\left(S_{\max }^{\mathrm{SPMT}}\right)$ that can be measured by each SPMT without saturating the acquisition electronics (since the sPMT linearity is maintained up to much larger particle density inside the WCD). Its average value $\left.\left(<S_{\max }^{\text {sPMT }}\right\rangle\right)$ is shown in Fig.6 (Left) for a set of stations where the sPMT high voltage has been manually set to roughly match the requirement of measuring signals up to 20,000 VEM without saturation. The dispersion among the daily estimations results to be of the order of few \% for all the analyzed stations, demonstrating the stability of the dynamic range extension when the temperature excursion from day to day is negligible (an average variation of 5 degrees is found in the considered period).

Using the small showers events and the cross-calibration result, the relation between the signals in the WCD and in the scintillator can be examined for each single tank even on a timescale of few weeks, as shown in Fig.6 (Right). The SSD signals are expressed in MIP while the WCD ones in VEM, with the WCD PMTs covering the dynamic range up to the saturation (blue dots) and the sPMT extending it up to a few $10^{4}$ VEM (red dots). The signals show a larger dispersion with respect to standard ultra-high energy air showers. Indeed the vast majority of these small showers are estimated to have energy lower than few $\mathrm{PeV}$, producing non uniform distributions of particles over the station and thus very different signals in the WCD and SSD. 

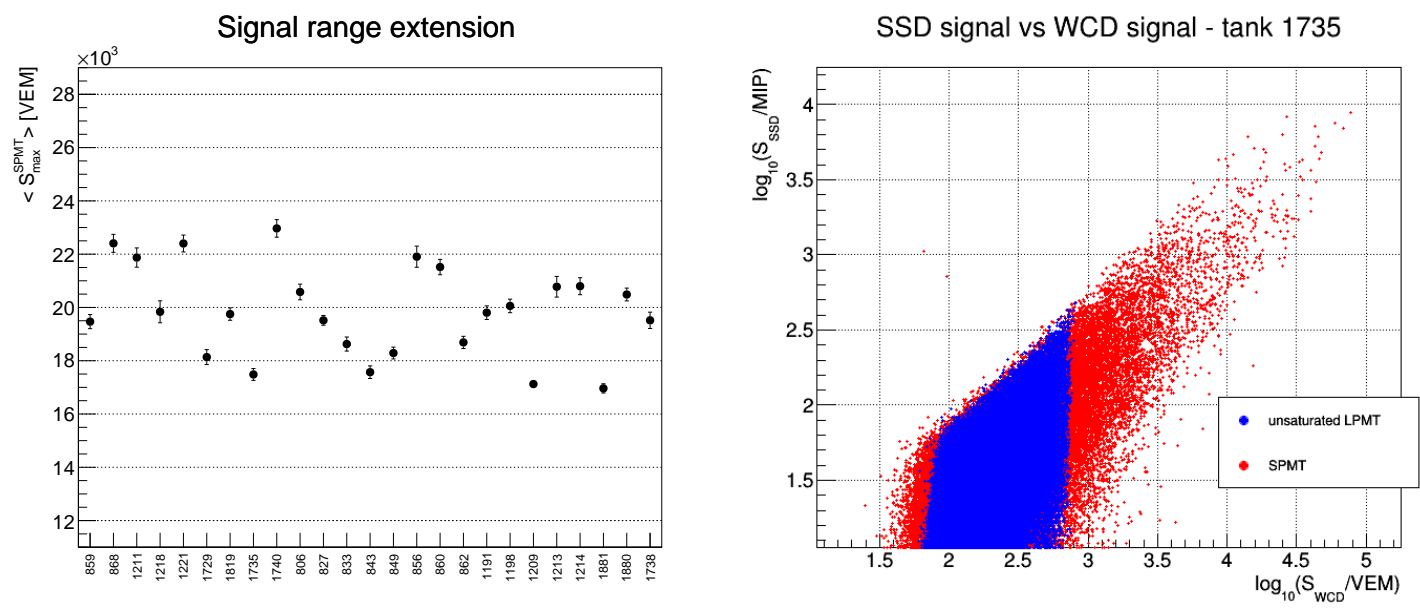

Figure 6: Left: Expected maximum sPMT signal without saturation for a subset of stations, estimated using 1 month of small showers data. The error bars in each value represent the dispersion among the daily estimations of $S_{\max }^{\mathrm{SPMT}}$. Right: Correlation between the WCD and the scintillator signals for one upgraded AugerPrime station, using the small showers selected for the cross-calibration procedure.

\section{Conclusions}

AugerPrime is in its commissioning phase and all detectors are being installed in the field. The UUBs are in production. All the phases have been intensively verified and tested and the process is now in its full active phase. Already 81 boards are in Argentina and at the end of July 202184 more will be sent and 400 more are planned at the end of August 2021. Since December 2020, all UUBs in the field are included in the standard DAQ and monitored offline. The performances of the boards are stable and well within the design requirements. The process is going on as expected and we foreseen the completion of the UUB deployment by the end of 2022. Special thanks are due to the local staff involved in the operations.

\section{References}

[1] A. Aab et al. [Pierre Auger Coll.], The Pierre Auger Cosmic Ray Observatory, Nucl. Instrum. Meth. A 798, $172-213$ (2015) [1502.01323].

[2] A. Aab et al. [for the Pierre Auger Coll.], The Pierre Auger Observatory Upgrade, [1604 . 03637].

[3] G. Cataldi [for the Pierre Auger Coll.], The upgrade of the Pierre Auger Observatory with the Scintillator Surface Detector, These Proceedings, PoS(ICRC 2021) 1017.

[4] A. Castellina [for the Pierre Auger Coll.], AugerPrime: the Pierre Auger Observatory Upgrade, EPJ Web Conf. 210 (2019) 06002, [1905.04472].

[5] A. Botti [for the Pierre Auger Coll.], Status and performance of the underground muon detector of the Pierre Auger Observatory, These Proceedings, PoS(ICRC 2021) 778.

[6] F. Schlüter, [for the Pierre Auger Coll.], Expected performance of the AugerPrime Radio Detector, In this Proceedings, PoS(ICRC 2021) 1210.

[7] D.F. Nitz [for the Pierre Auger Coll.], New Electronics for the Surface Detectors of the Pierre Auger Observatory, Proc. of the 36th Int. Cosmic Ray Conf., PoS(ICRC 2019) 370.

[8] M. Bohacova [for the Pierre Auger Coll.], Test benches for the upgrade of the Pierre Auger Observatory electronics, Proc. of the 36th Int. Cosmic Ray Conf., PoS(ICRC 2019) 199. 


\section{The Pierre Auger Collaboration}

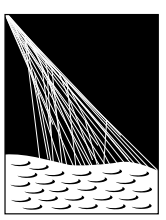

PIERRE

AUSGERVATORY

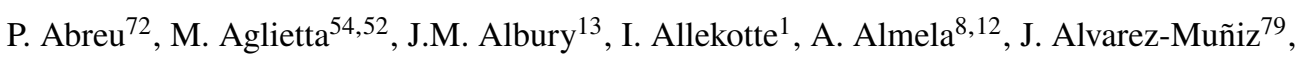
R. Alves Batista ${ }^{80}$, G.A. Anastasi ${ }^{63,52}$, L. Anchordoqui ${ }^{87}$, B. Andrada ${ }^{8}$, S. Andringa ${ }^{72}$, C. $\mathrm{Aramo}^{50}$, P.R. Araújo Ferreira ${ }^{42}$, J. C. Arteaga Velázquez ${ }^{67}$, H. Asorey $^{8}$, P. Assis ${ }^{72}$, G. Avila ${ }^{11}$, A.M. Badescu ${ }^{75}$, A. Bakalova ${ }^{32}$, A. Balaceanu ${ }^{73}$, F. Barbato ${ }^{45,46}$, R.J. Barreira Luz $^{72}$, K.H. Becker ${ }^{38}$, J.A. Bellido ${ }^{13,69}$, C. Berat ${ }^{36}$, M.E. Bertaina ${ }^{63,52}$, X. Bertou ${ }^{1}$, P.L. Biermann ${ }^{b}$, V. Binet ${ }^{6}$, K. Bismark ${ }^{39,8}$, T. Bister ${ }^{42}$, J. Biteau ${ }^{37}$, J. Blazek ${ }^{32}$, C. Bleve ${ }^{36}$, M. Boháčová ${ }^{32}$, D. Boncioli ${ }^{57,46}$, C. Bonifazi ${ }^{9,26}$, L. Bonneau Arbeletche ${ }^{21}$, N. Borodai ${ }^{70}$, A.M. Botti ${ }^{8}$, J. Brack ${ }^{d}$, T. Bretz ${ }^{42}$, P.G. Brichetto Orchera ${ }^{8}$, F.L. Briechle ${ }^{42}$, P. Buchholz ${ }^{44}$, A. Bueno ${ }^{78}$, S. Buitink ${ }^{15}$, M. Buscemi ${ }^{47}$, M. Büsken ${ }^{39,8}$, K.S. Caballero-Mora ${ }^{66}$, L. Caccianiga ${ }^{59,49}$, F. Canfora ${ }^{80,81}$, I. Caracas ${ }^{38}$, J.M. Carceller ${ }^{78}$, R. Caruso ${ }^{58,47}$, A. Castellina ${ }^{54,52}$, F. Catalanii ${ }^{19}$, G. Cataldi ${ }^{48}$, L. Cazon ${ }^{72}$, M. Cerda ${ }^{10}$, J.A. Chinellato ${ }^{22}$, J. Chudoba ${ }^{32}$, L. Chytka ${ }^{33}$, R.W. Clay ${ }^{13}$, A.C. Cobos Ceruttii ${ }^{7}$, R. Colalillo ${ }^{60,50}$, A. Coleman ${ }^{93}$, M.R. Coluccia ${ }^{48}$, R. Conceição ${ }^{72}$, A. Condorelli ${ }^{45,46}$, G. Consolati ${ }^{49,55}$, F. Contreras ${ }^{11}$, F. Convenga ${ }^{56,48}$, D. Correia dos Santos $^{28}$, C.E. Covault ${ }^{85}$, S. Dasso ${ }^{5,3}$, K. Daumiller ${ }^{41}$, B.R. Dawson ${ }^{13}$, J.A. Day ${ }^{13}$, R.M. de Almeida $^{28}$, J. de Jesús ${ }^{8,41}$, S.J. de Jong ${ }^{80,81}$, G. De Mauro ${ }^{80,81}$, J.R.T. de Mello Neto ${ }^{26,27}$, I. De Mitri ${ }^{45,46}$, J. de Oliveira ${ }^{18}$, D. de Oliveira Franco ${ }^{22}$, F. de Palma ${ }^{56,48}$, V. de Souza $^{20}$, E. De Vito ${ }^{56,48}$, M. del Río ${ }^{11}$, O. Deligny ${ }^{34}$, L. Deval ${ }^{41,8}$, A. di Matteo $^{52}$, C. Dobrigkeit ${ }^{22}$, J.C. D’Olivo ${ }^{68}$, L.M. Domingues Mendes ${ }^{72}$, R.C. dos Anjos ${ }^{25}$, D. dos Santos $^{28}$, M.T. Dova ${ }^{4}$, J. Ebr ${ }^{32}$, R. Engel ${ }^{39,41}$, I. Epicoco ${ }^{56,48}$, M. Erdmann ${ }^{42}$, C.O. Escobar ${ }^{a}$, A. Etchegoyen ${ }^{8,12}$, H. Falcke ${ }^{80,82,81}$, J. Farmer ${ }^{92}$, G. Farrar ${ }^{90}$, A.C. Fauth ${ }^{22}$, N. Fazzini ${ }^{a}$, F. Feldbusch ${ }^{40}$, F. Fenu ${ }^{54,52}$,

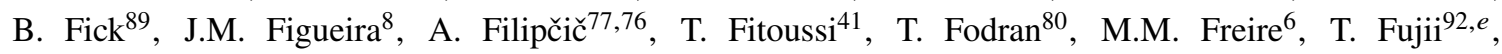
A. Fuster $^{8,12}$, C. Galea ${ }^{80}$, C. Galelli ${ }^{59,49}$, B. García ${ }^{7}$, A.L. Garcia Vegas ${ }^{42}$, H. Gemmeke ${ }^{40}$, F. Gesualdi ${ }^{8,41}$, A. Gherghel-Lascu ${ }^{73}$, P.L. Ghia ${ }^{34}$, U. Giaccari ${ }^{80}$, M. Giammarchi ${ }^{49}$, J. Glombitza ${ }^{42}$, F. Gobbi ${ }^{10}$, F. Gollan ${ }^{8}$, G. Golup ${ }^{1}$, M. Gómez Berisso ${ }^{1}$, P.F. Gómez Vitale ${ }^{11}$, J.P. Gongora ${ }^{11}$, J.M. González ${ }^{1}$, N. González ${ }^{14}$, I. Goos ${ }^{1,41}$, D. Góra ${ }^{70}$, A. Gorgi ${ }^{54,52}$, M. Gottowik ${ }^{38}$, T.D. Grubb ${ }^{13}$, F. Guarino ${ }^{60,50}$, G.P. Guedes ${ }^{23}$, E. Guido ${ }^{52,63}$, S. $\mathrm{Hahn}^{41,8}$, P. $\mathrm{Hamal}^{32}$, M.R. Hampel ${ }^{8}$, P. Hansen ${ }^{4}$, D. Harari ${ }^{1}$, V.M. Harvey ${ }^{13}$, A. Haungs ${ }^{41}$, T. Hebbeker ${ }^{42}$, D. Heck ${ }^{41}$, G.C. Hill ${ }^{13}$, C. Hojvat ${ }^{a}$, J.R. Hörandel ${ }^{80,81}$, P. Horvath ${ }^{33}$, M. Hrabovský ${ }^{33}$, T. Huege ${ }^{41,15}$, A. Insolia ${ }^{58,47}$, P.G. Isar $^{74}$, P. Janecek ${ }^{32}$, J.A. Johnsen ${ }^{86}$, J. Jurysek ${ }^{32}$, A. Kääpä ${ }^{38}$, K.H. Kampert ${ }^{38}$, N. Karastathis ${ }^{41}$, B. Keilhauer ${ }^{41}$, J. Kemp ${ }^{42}$, A. Khakurdikar ${ }^{80}$, V.V. Kizakke Covilakam $^{8,41}$, H.O. Klages ${ }^{41}$, M. Kleifges ${ }^{40}$, J. Kleinfeller ${ }^{10}$, M. Köpke ${ }^{39}$, N. Kunka ${ }^{40}$, B.L. Lago ${ }^{17}$, R.G. Lang ${ }^{20}$, N. Langner ${ }^{42}$, M.A. Leigui de Oliveira ${ }^{24}$, V. Lenok $^{41}$, A. Letessier-Selvon ${ }^{35}$, I. LhenryYvon $^{34}$, D. Lo Presti ${ }^{58,47}$, L. Lopes ${ }^{72}$, R. López ${ }^{64}$, L. Lu ${ }^{94}$, Q. Luce ${ }^{39}$, J.P. Lundquist ${ }^{76}$, A. Machado Payeras $^{22}$, G. Mancarella ${ }^{56,48}$, D. Mandat ${ }^{32}$, B.C. Manning ${ }^{13}$, J. Manshanden ${ }^{43}$, P. Mantsch ${ }^{a}$, S. Marafico ${ }^{34}$, A.G. Mariazzi ${ }^{4}$, I.C. Mariş ${ }^{14}$, G. Marsella ${ }^{61,47}$, D. Martello ${ }^{56,48}$, S. Martinelli ${ }^{41,8}$, O. Martínez Bravo ${ }^{64}$, M. Mastrodicasa ${ }^{57,46}$, H.J. Mathes ${ }^{41}$, J. Matthews ${ }^{88}$, G. Matthiae ${ }^{62,51}$, E. Mayotte ${ }^{38}$, P.O. Mazur ${ }^{a}$, G. MedinaTanco $^{68}$, D. Melo $^{8}$, A. Menshikov ${ }^{40}$, K.-D. Merenda ${ }^{86}$, S. Michal ${ }^{33}$, M.I. Micheletti ${ }^{6}$, L. Miramonti ${ }^{59,49}$, S. Mollerach ${ }^{1}$, F. Montanet ${ }^{36}$, C. Morello ${ }^{54,52}$, M. Mostafá ${ }^{91}$, A.L. Müller ${ }^{8}$, M.A. Muller ${ }^{22}$, K. Mulrey ${ }^{15}$, R. Mussa ${ }^{52}$, M. Muzio ${ }^{90}$, W.M. Namasaka ${ }^{38}$, A. Nasr-Esfahani ${ }^{38}$, L. Nellen ${ }^{68}$, M. Niculescu-Oglinzanu ${ }^{73}$, M. Niechciol ${ }^{44}$, D. Nitz ${ }^{89}$, D. Nosek ${ }^{31}$, V. Novotny ${ }^{31}$, L. Nožka ${ }^{33}$, A Nucita ${ }^{56,48}$, L.A. Núñez ${ }^{30}$, M. Palatka ${ }^{32}$, J. Pallotta ${ }^{2}$, P. Papenbreer ${ }^{38}$, G. Parente ${ }^{79}$, A. Parra ${ }^{64}$, J. Pawlowsky ${ }^{38}$, M. Pech ${ }^{32}$, F. Pedreira ${ }^{79}$, J. Pȩkala ${ }^{70}$, R. Pelayo ${ }^{65}$, J. Peña-Rodriguez ${ }^{30}$, E.E. Pereira Martins ${ }^{39,8}$, J. Perez Armand ${ }^{21}$, C. Pérez Bertolli $^{8,41}$, M. Perlin ${ }^{8,41}$, L. Perrone ${ }^{56,48}$, S. Petrera ${ }^{45,46}$, T. Pierog ${ }^{41}$, M. Pimenta ${ }^{72}$, V. Pirronello ${ }^{58,47}$, M. Platino ${ }^{8}$, B. Pont $^{80}$, M. Pothast ${ }^{81,80}$, P. Privitera ${ }^{92}$, M. Prouza ${ }^{32}$, A. Puyleart ${ }^{89}$, S. Querchfeld ${ }^{38}$, J. Rautenberg ${ }^{38}$, D. Ravignani ${ }^{8}$, M. Reininghaus ${ }^{41,8}$, J. Ridky ${ }^{32}$, F. Riehn ${ }^{72}$, M. Risse ${ }^{44}$, V. Rizi ${ }^{57,46}$, W. Rodrigues de Carvalho ${ }^{21}$, J. Rodriguez Rojo ${ }^{11}$, M.J. Roncoroni ${ }^{8}$, S. Rossoni ${ }^{43}$, M. Roth ${ }^{41}$, E. Roulet ${ }^{1}$, A.C. Rovero ${ }^{5}$, P. Ruehl ${ }^{44}$, A. Saftoiu ${ }^{73}$, F. Salamida ${ }^{57,46}$, H. Salazar ${ }^{64}$, G. Salina ${ }^{51}$, J.D. Sanabria Gomez ${ }^{30}$, F. Sánchez ${ }^{8}$, E.M. Santos ${ }^{21}$, E. Santos ${ }^{32}$, F. Sarazin ${ }^{86}$, R. Sarmento ${ }^{72}$, C. Sarmiento-Cano ${ }^{8}$, R. Sato ${ }^{11}$, 
P. Savina ${ }^{56,48,34,94}$, C.M. Schäfer ${ }^{41}$, V. Scherini ${ }^{56,48}$, H. Schieler ${ }^{41}$, M. Schimassek ${ }^{39,8}$, M. Schimp ${ }^{38}$, F. Schlüter ${ }^{41,8}$, D. Schmidt ${ }^{39}$, O. Scholten ${ }^{84,15}$, P. Schovánek ${ }^{32}$, F.G. Schröder ${ }^{93,41}$, S. Schröder ${ }^{38}$, J. Schulte ${ }^{42}$, S.J. Sciutto ${ }^{4}$, M. Scornavacche ${ }^{8,41}$, A. Segreto ${ }^{53,47}$, S. Sehgal ${ }^{38}$, R.C. Shellard ${ }^{16}$, G. Sigl ${ }^{43}$, G. Silli ${ }^{8,41}$, O. Sima ${ }^{73, f}$, R. Šmída ${ }^{92}$, P. Sommers ${ }^{91}$, J.F. Soriano ${ }^{87}$, J. Souchard ${ }^{36}$, R. Squartini ${ }^{10}$, M. Stadelmaier ${ }^{41,8}$, D. Stanca ${ }^{73}$, S. Staničc ${ }^{76}$, J. Stasielak ${ }^{70}$, P. Stassi ${ }^{36}$, A. Streich ${ }^{39,8}$, M. Suárez-Durán ${ }^{14}$, T. Sudholz ${ }^{13}$, T. Suomijärvi ${ }^{37}$, A.D. Supanitsky ${ }^{8}$, Z. Szadkowski ${ }^{71}$, A. Tapia ${ }^{29}$, C. Taricco ${ }^{63,52}$, C. Timmermans ${ }^{81,80}$, O. Tkachenko ${ }^{41}$, P. Tobiska ${ }^{32}$, C.J. Todero Peixoto ${ }^{19}$, B. Tomé ${ }^{72}$, Z. Torrès ${ }^{36}$, A. Travaini ${ }^{10}$, P. Travnicek ${ }^{32}$, C. Trimarelli ${ }^{57,46}$, M. Tueros ${ }^{4}$, R. Ulrich ${ }^{41}$, M. Unger ${ }^{41}$, L. Vaclavek ${ }^{33}$, M. Vacula ${ }^{33}$, J.F. Valdés Galicia ${ }^{68}$, L. Valore ${ }^{60,50}$, E. Varela ${ }^{64}$, A. Vásquez-Ramírez ${ }^{30}$, D. Veberič ${ }^{41}$, C. Ventura ${ }^{27}$, I.D. Vergara Quispe ${ }^{4}$, V. Verzi ${ }^{51}$, J. Vicha ${ }^{32}$, J. Vink ${ }^{83}$, S. Vorobiov ${ }^{76}$, H. Wahlberg ${ }^{4}$, C. Watanabe ${ }^{26}$, A.A. Watson ${ }^{c}$, M. Weber ${ }^{40}$, A. Weindl ${ }^{41}$, L. Wiencke ${ }^{86}$, H. Wilczyński ${ }^{70}$, M. Wirtz ${ }^{42}$, D. Wittkowski ${ }^{38}$, B. Wundheiler ${ }^{8}$, A. Yushkov $^{32}$, O. Zapparrata ${ }^{14}$, E. Zas ${ }^{79}$, D. Zavrtanik ${ }^{76,77}$, M. Zavrtanik ${ }^{77,76}$, L. Zehrer ${ }^{76}$

${ }^{1}$ Centro Atómico Bariloche and Instituto Balseiro (CNEA-UNCuyo-CONICET), San Carlos de Bariloche, Argentina

${ }^{2}$ Centro de Investigaciones en Láseres y Aplicaciones, CITEDEF and CONICET, Villa Martelli, Argentina

${ }^{3}$ Departamento de Física and Departamento de Ciencias de la Atmósfera y los Océanos, FCEyN, Universidad de Buenos Aires and CONICET, Buenos Aires, Argentina

${ }^{4}$ IFLP, Universidad Nacional de La Plata and CONICET, La Plata, Argentina

${ }^{5}$ Instituto de Astronomía y Física del Espacio (IAFE, CONICET-UBA), Buenos Aires, Argentina

${ }^{6}$ Instituto de Física de Rosario (IFIR) - CONICET/U.N.R. and Facultad de Ciencias Bioquímicas y Farmacéuticas U.N.R., Rosario, Argentina

${ }^{7}$ Instituto de Tecnologías en Detección y Astropartículas (CNEA, CONICET, UNSAM), and Universidad Tecnológica Nacional - Facultad Regional Mendoza (CONICET/CNEA), Mendoza, Argentina

${ }^{8}$ Instituto de Tecnologías en Detección y Astropartículas (CNEA, CONICET, UNSAM), Buenos Aires, Argentina

${ }^{9}$ International Center of Advanced Studies and Instituto de Ciencias Físicas, ECyT-UNSAM and CONICET, Campus Miguelete - San Martín, Buenos Aires, Argentina

${ }^{10}$ Observatorio Pierre Auger, Malargüe, Argentina

${ }^{11}$ Observatorio Pierre Auger and Comisión Nacional de Energía Atómica, Malargüe, Argentina

12 Universidad Tecnológica Nacional - Facultad Regional Buenos Aires, Buenos Aires, Argentina

${ }^{13}$ University of Adelaide, Adelaide, S.A., Australia

14 Université Libre de Bruxelles (ULB), Brussels, Belgium

15 Vrije Universiteit Brussels, Brussels, Belgium

${ }^{16}$ Centro Brasileiro de Pesquisas Fisicas, Rio de Janeiro, RJ, Brazil

${ }^{17}$ Centro Federal de Educação Tecnológica Celso Suckow da Fonseca, Nova Friburgo, Brazil

${ }^{18}$ Instituto Federal de Educação, Ciência e Tecnologia do Rio de Janeiro (IFRJ), Brazil

${ }^{19}$ Universidade de São Paulo, Escola de Engenharia de Lorena, Lorena, SP, Brazil

${ }^{20}$ Universidade de São Paulo, Instituto de Física de São Carlos, São Carlos, SP, Brazil

${ }^{21}$ Universidade de São Paulo, Instituto de Física, São Paulo, SP, Brazil

${ }^{22}$ Universidade Estadual de Campinas, IFGW, Campinas, SP, Brazil

${ }^{23}$ Universidade Estadual de Feira de Santana, Feira de Santana, Brazil

${ }^{24}$ Universidade Federal do ABC, Santo André, SP, Brazil

${ }^{25}$ Universidade Federal do Paraná, Setor Palotina, Palotina, Brazil

${ }^{26}$ Universidade Federal do Rio de Janeiro, Instituto de Física, Rio de Janeiro, RJ, Brazil

${ }^{27}$ Universidade Federal do Rio de Janeiro (UFRJ), Observatório do Valongo, Rio de Janeiro, RJ, Brazil

${ }^{28}$ Universidade Federal Fluminense, EEIMVR, Volta Redonda, RJ, Brazil

${ }^{29}$ Universidad de Medellín, Medellín, Colombia

${ }^{30}$ Universidad Industrial de Santander, Bucaramanga, Colombia

${ }^{31}$ Charles University, Faculty of Mathematics and Physics, Institute of Particle and Nuclear Physics, Prague, Czech Republic

32 Institute of Physics of the Czech Academy of Sciences, Prague, Czech Republic 
${ }^{33}$ Palacky University, RCPTM, Olomouc, Czech Republic

34 CNRS/IN2P3, IJCLab, Université Paris-Saclay, Orsay, France

${ }^{35}$ Laboratoire de Physique Nucléaire et de Hautes Energies (LPNHE), Sorbonne Université, Université de Paris, CNRSIN2P3, Paris, France

${ }^{36}$ Univ. Grenoble Alpes, CNRS, Grenoble Institute of Engineering Univ. Grenoble Alpes, LPSC-IN2P3, 38000 Grenoble, France

${ }^{37}$ Université Paris-Saclay, CNRS/IN2P3, IJCLab, Orsay, France

38 Bergische Universität Wuppertal, Department of Physics, Wuppertal, Germany

${ }^{39}$ Karlsruhe Institute of Technology (KIT), Institute for Experimental Particle Physics, Karlsruhe, Germany

${ }^{40}$ Karlsruhe Institute of Technology (KIT), Institut für Prozessdatenverarbeitung und Elektronik, Karlsruhe, Germany

${ }^{41}$ Karlsruhe Institute of Technology (KIT), Institute for Astroparticle Physics, Karlsruhe, Germany

${ }^{42}$ RWTH Aachen University, III. Physikalisches Institut A, Aachen, Germany

${ }^{43}$ Universität Hamburg, II. Institut für Theoretische Physik, Hamburg, Germany

44 Universität Siegen, Department Physik - Experimentelle Teilchenphysik, Siegen, Germany

${ }^{45}$ Gran Sasso Science Institute, L'Aquila, Italy

46 INFN Laboratori Nazionali del Gran Sasso, Assergi (L'Aquila), Italy

${ }^{47}$ INFN, Sezione di Catania, Catania, Italy

${ }^{48}$ INFN, Sezione di Lecce, Lecce, Italy

${ }^{49}$ INFN, Sezione di Milano, Milano, Italy

${ }^{50}$ INFN, Sezione di Napoli, Napoli, Italy

${ }^{51}$ INFN, Sezione di Roma "Tor Vergata", Roma, Italy

52 INFN, Sezione di Torino, Torino, Italy

53 Istituto di Astrofisica Spaziale e Fisica Cosmica di Palermo (INAF), Palermo, Italy

54 Osservatorio Astrofisico di Torino (INAF), Torino, Italy

55 Politecnico di Milano, Dipartimento di Scienze e Tecnologie Aerospaziali , Milano, Italy

56 Università del Salento, Dipartimento di Matematica e Fisica "E. De Giorgi”, Lecce, Italy

57 Università dell'Aquila, Dipartimento di Scienze Fisiche e Chimiche, L'Aquila, Italy

58 Università di Catania, Dipartimento di Fisica e Astronomia, Catania, Italy

${ }^{59}$ Università di Milano, Dipartimento di Fisica, Milano, Italy

${ }^{60}$ Università di Napoli "Federico II", Dipartimento di Fisica "Ettore Pancini”, Napoli, Italy

${ }^{61}$ Università di Palermo, Dipartimento di Fisica e Chimica "E. Segrè", Palermo, Italy

62 Università di Roma "Tor Vergata", Dipartimento di Fisica, Roma, Italy

${ }^{63}$ Università Torino, Dipartimento di Fisica, Torino, Italy

${ }^{64}$ Benemérita Universidad Autónoma de Puebla, Puebla, México

${ }^{65}$ Unidad Profesional Interdisciplinaria en Ingeniería y Tecnologías Avanzadas del Instituto Politécnico Nacional (UPIITA-IPN), México, D.F., México

66 Universidad Autónoma de Chiapas, Tuxtla Gutiérrez, Chiapas, México

${ }^{67}$ Universidad Michoacana de San Nicolás de Hidalgo, Morelia, Michoacán, México

${ }^{68}$ Universidad Nacional Autónoma de México, México, D.F., México

${ }^{69}$ Universidad Nacional de San Agustin de Arequipa, Facultad de Ciencias Naturales y Formales, Arequipa, Peru

${ }^{70}$ Institute of Nuclear Physics PAN, Krakow, Poland

${ }^{71}$ University of Łódź, Faculty of High-Energy Astrophysics, Łódź, Poland

${ }^{72}$ Laboratório de Instrumentação e Física Experimental de Partículas - LIP and Instituto Superior Técnico - IST, Universidade de Lisboa - UL, Lisboa, Portugal

73 "Horia Hulubei” National Institute for Physics and Nuclear Engineering, Bucharest-Magurele, Romania

${ }^{74}$ Institute of Space Science, Bucharest-Magurele, Romania

75 University Politehnica of Bucharest, Bucharest, Romania

76 Center for Astrophysics and Cosmology (CAC), University of Nova Gorica, Nova Gorica, Slovenia

${ }^{77}$ Experimental Particle Physics Department, J. Stefan Institute, Ljubljana, Slovenia

78 Universidad de Granada and C.A.F.P.E., Granada, Spain

${ }^{79}$ Instituto Galego de Física de Altas Enerxías (IGFAE), Universidade de Santiago de Compostela, Santiago de Compostela, Spain 
${ }^{80}$ IMAPP, Radboud University Nijmegen, Nijmegen, The Netherlands

${ }^{81}$ Nationaal Instituut voor Kernfysica en Hoge Energie Fysica (NIKHEF), Science Park, Amsterdam, The Netherlands

82 Stichting Astronomisch Onderzoek in Nederland (ASTRON), Dwingeloo, The Netherlands

${ }^{83}$ Universiteit van Amsterdam, Faculty of Science, Amsterdam, The Netherlands

${ }^{84}$ University of Groningen, Kapteyn Astronomical Institute, Groningen, The Netherlands

85 Case Western Reserve University, Cleveland, OH, USA

${ }^{86}$ Colorado School of Mines, Golden, CO, USA

${ }^{87}$ Department of Physics and Astronomy, Lehman College, City University of New York, Bronx, NY, USA

${ }^{88}$ Louisiana State University, Baton Rouge, LA, USA

${ }^{89}$ Michigan Technological University, Houghton, MI, USA

${ }^{90}$ New York University, New York, NY, USA

${ }^{91}$ Pennsylvania State University, University Park, PA, USA

92 University of Chicago, Enrico Fermi Institute, Chicago, IL, USA

93 University of Delaware, Department of Physics and Astronomy, Bartol Research Institute, Newark, DE, USA

94 University of Wisconsin-Madison, Department of Physics and WIPAC, Madison, WI, USA

${ }^{a}$ Fermi National Accelerator Laboratory, Fermilab, Batavia, IL, USA

${ }^{b}$ Max-Planck-Institut für Radioastronomie, Bonn, Germany

${ }^{c}$ School of Physics and Astronomy, University of Leeds, Leeds, United Kingdom

${ }^{d}$ Colorado State University, Fort Collins, CO, USA

$e^{e}$ now at Hakubi Center for Advanced Research and Graduate School of Science, Kyoto University, Kyoto, Japan

$f$ also at University of Bucharest, Physics Department, Bucharest, Romania 